\title{
The protective action of tocopherol and acetylsalicylic acid on the behavior of rats treated with dioxins
}

\author{
Joanna Rosińczuk ${ }^{1, A-F}$, Robert Dymarek ${ }^{1, C-F}$, Ireneusz Całkosiński 2, A-F \\ ${ }^{1}$ Department of Nervous System Diseases, Wroclaw Medical University, Poland \\ ${ }^{2}$ Independent Laboratory of Clinical Neurotoxicology and Environmental Diagnostics, Wroclaw Medical University, Poland \\ A - research concept and design; B - collection and/or assembly of data; C - data analysis and interpretation; \\ $D$ - writing the article; $E$ - critical revision of the article; $F$ - final approval of the article
}

\author{
Address for correspondence \\ Joanna Rosińczuk \\ E-mail:joanna.rosinczuk@umed.wroc.pl \\ Funding sources \\ None declared

\section{Conflict of interest} \\ None declared
}

Received on February 11, 2016

Reviewed on June 29, 2016

Accepted on November 23, 2016

DOI

$10.17219 /$ acem/67314

\section{Copyright}

Copyright by Author(s)

This is an article distributed under the terms of the

Creative Commons Attribution Non-Commercial License

(http://creativecommons.org/licenses/by-nc-nd/4.0/)

\begin{abstract}
Background. Dioxins contribute to neurological disorders in humans and animals, causing also neurological disorders in offspring during prenatal and postnatal periods. These compounds significantly affect the development of the central nervous system (CNS) structures, which results in behavioral changes. Tocopherol (TCP) and acetylsalicylic acid (ASA) may provide protective measures to reduce the inflammatory effects in the CNS associated with free radicals generated by 2,3,7,8-tetrachlorodibenzo-p-dioxin (TCDD), thus contributing to the reduction of the negative effects of dioxin.
\end{abstract}

Objectives. The main objective of this study was to determine the influence of dioxin on rats and their behavioral functions, and to ascertain whether a combined administration of TCP and ASA to rats treated with TCDD shows the possibility of potential protective effect on the functioning of the CNS.

Material and methods. Experiments were performed on 75 female and 12 male Buffalo strain rats, which are offspring of females from particular study groups. TCDD was used in the experiments, TCP and ASA were administered orally every day for 3 weeks. Animals were subjected to behavioral testing: the tail and swimming tests.

Results. During the observation of the offspring of both sexes born to females exposed to TCDD, males did not demonstrate any attempt to swim, whereas in females, the immobility time was significantly extended. Assessing the response times from the tail test in the animals treated with dioxins in relation to the control group, it was demonstrated that the response time was extended in the $3^{\text {rd }}$ measurement in both females and males.

Conclusions. Dioxin is characterized by neurotoxic effect causing behavioral disorders associated with prolonged response times. The use of TCP after the administration of dioxins causes a significant reduction and improvement of reflex response times. In contrast, ASA reduces the reflex response times also in the offspring of females exposed to TCDD and ASA.

Key words: central nervous system, 2,3,7,8-tetrachlorodibenzo-p-dioxin, tocopherol, acetylsalicylic acid, behavioral functions 


\section{Introduction}

Dioxins due to lipophilicity are mostly deposited in adipose tissue and nerve tissue. The rationale for the study was to ascertain depression in patients intoxicated with dioxins, treated with antidepressants. ${ }^{1}$ In experimental studies on animals treated with 2,3,7,8-tetrachlorodibenzo-p-dioxin (TCDD), behavioral disorders connected with reduced activity and apathy were observed. ${ }^{2-6}$

Dioxin and related compounds are the cause of neurological disorders in humans and experimental animals as well as their offspring in the perinatal period after exposure to these compounds through the placenta and milk in prenatal and postnatal periods. ${ }^{7-9}$ Dioxins significantly affect brain development in the prenatal and postnatal periods associated with the time of breastfeeding by mothers intoxicated with dioxins. ${ }^{10-13}$ Changes caused by dioxins in the central nervous system (CNS) in pre- and postnatal periods lead to later changes in life. Functional disorders of the brain structures, including the cerebellum and diencephalon, should also be associated with changes in thyroid hormone levels and estrogen receptors (ER) induced by dioxins. ${ }^{9,14-17}$

It was shown that dioxins lead to the underdevelopment of the bones and teeth in both humans and animals as a result of mineralization disorders. ${ }^{18-20}$ It was found that after birth there is a significant concentration of dioxins in the breast milk, correlating negatively with a newborn head circumference. This indicates that the brain development of the fetus can be significantly affected by maternal exposure to TCDD, which, together with milk, provides a substantial amount of dioxins to the offspring. ${ }^{13,21,22}$ In patients intoxicated with dioxins, there were observed: severe depression, apathy and reduced physical activity. ${ }^{23,24}$ This should be associated with the anti-estrogenic effects of TCDD. ${ }^{25}$ Experimental studies in animals treated with TCDD by other authors also found behavioral disorders associated with decreased motility, apathy and circadian rhythm disruption. ${ }^{26-28}$ Studies in rainbow trout revealed that exposure to TCDD induces biochemical and structural changes in the eye and brain, leading to behavioral deficits and reduced efficiency. ${ }^{29}$

Tocopherol (TCP) reduces the contact of TCDD with the aryl hydrocarbon receptor (AhR) blocking the formation of $C Y P 1 A 1$, thereby reducing the amount of generated free radicals. In this case, a TCP beneficial effect is manifested in selected biochemical indicators of the rats blood exposed to TCDD in which TCP is used in high doses. ${ }^{14,30,31}$ According to Hassoun et al., there are areas in the brain which are less or more reactive to the active oxygen species. ${ }^{32,33}$ Areas sensitive to the effects of free radicals generated by TCDD include the cerebral cortex, hippocampus, cerebellum, and brain stem. It was shown that administration of vitamin $\mathrm{E}$ reduced the secretion of reactive oxygen species (ROS) induced by TCDD; at the same time it showed the protective properties of the brain structures. ${ }^{34,35}$ A significant increase in tumor necrosis factor alpha (TNF- $\alpha$ ) in mice treated with TCDD was also noted. In turn, the increase in the level of TNF- $\alpha$ is responsible for lethargy and anorexia. In experiments where high doses of TCP were used, the concentration of TNF- $\alpha$ significantly decreased, regardless of experimentally induced pleuritis. ${ }^{11,14,31}$

Acetylsalicylic acid (ASA), in addition to the antiprostaglandin effect in inflammatory reactions, decreases the production of free radicals, nitric oxide synthesis, and inhibits the production of proinflammatory cytokines such as TNF, IL-1, IL-6 through the inhibition of nuclear factor kappa $\beta$ (NF-kB). ASA also blocks COX-1 and COX-2. ${ }^{36-38}$ It was found that ASA also protects the brain structures against ROS. A study conducted by Maharaj et al. confirmed that the use of ASA in a daily dose of $100 \mathrm{mg}$ per $1 \mathrm{~kg}$ body weight (b.w.) inhibited the production of superoxide anions and lipid peroxidation products, thereby protecting the hippocampal neurons of a rat. ${ }^{39}$ The studies by McDonald et al. showed that salicylamide reduces the binding of TCDD to cytosolic AhR. ${ }^{37,38}$ It is concluded that this agent is a potent inhibitor of AhR and it blocks signal transduction initiated by the exposure to TCDD. The reason for the use of ASA as a protective substance in dioxin intoxication may be supported by the results of other studies, which found that ASA through the release of glutamate from nerve terminals has a presynaptic effect in the tail test, associated with the reduction of subsequent times. This leads to the phenomenon known as facilitation associated with facilitating neuromuscular transmission, and thus leading to faster reflex responses. ${ }^{40}$

The aim of this study was to determine the effect of dioxins on rats behavioral functions and to check whether the combined administration of TCP and ASA to rats treated with TCDD shows the potential of protective action of these compounds on the functioning of CNS in relation to the effects achieved by a single application of each of these compounds in the evaluation with the use of behavioral testing.

\section{Material and methods}

\section{Animals}

Experiments were performed on 117 rats: 75 females and 12 males of Buffalo strain, weighing 140-160 g, 8-10 weeks of age, and 30 females aged 6 weeks and weighing $120 \mathrm{~g}$ which were offspring of females from particular study groups listed below. All animals received humane care in compliance with the Guide for the Care and Use of Laboratory Animals (NRC 2011). All experiments were performed according to guidelines for the experimentation on animals. The study was approved by the Local Ethics Council for Animal Experiments (No. 38/2009). Animals were housed in air-conditioned rooms characterized 
by 15 air changes per hour at a temperature of $22^{\circ} \mathrm{C}$, humidity of $55 \%$ and a $12 / 12$ cycle of daylight. The rats were kept in polystyrene cages (6/cage), with free access to water and nutritious food Labofeed $\mathrm{H}$ dedicated for laboratory and breeding animals.

\section{TCDD administration}

In the experiments, 2,3,7,8-tetra-p-dioxin (Sigma Chemical Co. - Sigma-Aldrich Ltd., Poland) was used, administered at a dose of $5 \mu \mathrm{g} / \mathrm{mL}$ b.w. and $12.5 \mu \mathrm{g} / \mathrm{kg}$ b.w. dissolved in a $1 \%$ solution of dimethyl sulfoxide (DMSO) at a concentration of $1 \mu \mathrm{g} / \mathrm{mL}$. TCDD was administered intramuscularly in the hind limb muscles in a volume dependent on the body weight, i.e., $0.7-0.8 \mathrm{~mL}$, or $1.6-1.8 \mathrm{~mL}$ for administration of TCDD in a dose of $12.5 \mu \mathrm{g} / \mathrm{kg}$ b.w. The studies used $\alpha$-TCP acetate in an oily solution, administered daily for 3 weeks at a dose of $30 \mathrm{mg} / \mathrm{kg} \mathrm{b.w}$, subcutaneously (s.c.) in a volume of $0.2 \mathrm{~mL}$ and ASA administered orally (gavage) in the form of suspension in the starch solution at a dose of $50 \mathrm{mg} / \mathrm{kg}$ b.w., in a volume of $0.5 \mathrm{~mL}$ daily for 3 weeks. Induced pleurisy was induced with a single injection of $1 \%$ carrageenan solution into the pleural cavity between $5^{\text {th }}$ and $6^{\text {th }}$ right intercostal space in a volume of $0.15 \mathrm{~mL}$ in the experimental model worked out previously. ${ }^{41}$

\section{Behavioral tests}

Animals were subjected to behavioral tests, such as the tail test and the swimming test, performed in accordance with the applicable procedures. In both tests, the response time was measured with the use of calibrated metal electronic stopwatch Spokey Quary 2.

The swimming test involved placing a rat in a cylinder shaped container of $40 \mathrm{~cm}$ height and a diameter of $18 \mathrm{~cm}$ filled with water at $25^{\circ} \mathrm{C}$ for $5 \mathrm{~min}$. The aim of the study was to measure the time of immobility, i.e., the time that the test rat spends without any movement and to describe intensity of swimming scoring from 0 to 3 points: no swimming -0 points, intensive swimming -3 points.

The tail test consisted in placing the tail of the test subject in a plastic tube with a hole for a tail, which was then immersed in a glass container filled with water at a constant controlled temperature of $58^{\circ} \mathrm{C}$. The time which was measured was the time from the moment of tail insertion to its drawing from water by the rat. The longer the response time, the less energetic and active a rat was. To determine the dynamics of rat responses, the measures of response times in the tail test in each animal were performed 3 times at intervals of $60 \mathrm{~s}$.

The swimming test and the response time were performed in the following groups:

- $1 \mathrm{~A}$ - a control group C (F) of 6 females not exposed to effects of any agents;

- $1 \mathrm{~B}$ - a control group C (M) of 6 males not exposed to effects of any agents;
- 2 - TCDD group (F) of 6 females treated with TCDD (i.m.) 3 weeks prior to the study (F);

- 3 - TCDD group (M) of 6 males treated with TCDD (i.m.) 3 weeks prior to the study;

- 4 - TCDD + TCP group (F) of 6 females treated with TCDD (i.m.) and $\alpha$-TCP acetate 3 weeks prior to the study;

- 5 - TCDD + ASA group (F) of 6 females treated with TCDD (i.m.) and ASA 3 weeks prior to the study;

- $6-\mathrm{TCDD}+\mathrm{TCP}+\mathrm{ASA}$ group (F) of 6 females treated with TCDD (i.m.) 3 weeks prior to the study and daily with $\alpha$-TCP acetate and ASA;

- 7 - TCP group (F) of 6 females treated daily with $\alpha$-TCP acetate for the period of 3 weeks;

- 8-ASA group (F) of 6 females treated daily with ASA for the period of 3 weeks.

The tail test, which measured the response times, was also used in the study of 6 weeks old offspring born to females from the above groups:

- 1P - a control group PC of 6 randomly selected females from offspring born to 6 females from $C$ group $(F)$;

- 2P - PTCDD group of 6 randomly selected females from offspring born to 6 females from TCDD group (F);

- $3 P$ - PTCDD + TCP group of 6 randomly selected females from offspring born to 6 females from TCDD + TCP group $(\mathrm{F})$

- $4 \mathrm{P}-\mathrm{PTCDD}+\mathrm{ASA}$ group of 6 randomly selected females from offspring born to 6 females from TCDD + ASA group (F);

- 5P - PTCDD + TCP + ASA group of 6 randomly selected females from offspring born to 6 females from $\mathrm{TCDD}+\mathrm{TCP}+\mathrm{ASA}$ group $(\mathrm{F})$.

\section{Statistical analysis}

The data was processed with the use of EXEL spreadsheet and the package STATISTICA PL v. 9. The results of the swimming test measurements and response times in particular groups of the studied subjects were presented as mean values with standard deviations. The analysis of hypothesis on normality of distribution of variables was verified with the use of the Shapiro-Wilk test and DavidHellwig test at the significance level of 0.05 . The obtained values from the 2 subgroups were compared using the Student's t-test for unmatched pairs. The level of significance was set at $p=0.05$. The influence of one factor on the results of performed measurements was examined using the analysis of variance by repeated measures ANOVA. If the analysis of variance did not reveal the significance in differences between the analyzed mean values in subgroups, no further tests were carried out. When the null hypothesis of equality of means in subgroups was rejected in the analysis of variance $(p<0.05)$, the significance of differences between the means of particular subgroups was studied using post-hoc tests (multiple comparisons) - NIR test (least significant differences) and Duncan test. 
If the distribution of the studied parameter in one of the subgroups did not meet the assumptions of ANOVA, non-parametric alternative to ANOVA test was applied, the Kruskal-Wallis test.

\section{Results}

\section{Results of the tail test in adult subjects}

A comparison of subsequent response times in the control group $\mathrm{C}(\mathrm{F})$ to the response times in TCDD group (F) did not show statistically significant differences in the first 2 measurements; this difference occurred in the $3^{\text {rd }}$ measurement. The statistical evaluation of response times from the control group of females $C(F)$ showed that the response time evaluated with a tail test in 3 subsequent measurements did not change significantly. The reaction time in the control group $\mathrm{C}(\mathrm{M})$ of males slightly shortened with each measurement. In the $1^{\text {st }}$ measurement of response times in control groups $C(\mathrm{~F})$ and $C(\mathrm{M})$, there appears a statistically significant difference associated with a longer reaction time in males. In the $2^{\text {nd }}$ measurement, the response time in the group $\mathrm{C}(\mathrm{M})$ is longer than in $\mathrm{C}(\mathrm{F})$; however, this difference is not statistically significant. Mean response times for the control group of females and males $C(M)$ and $C(F)$ showed no statistically significant difference (Fig. 1, 2).
A comparison of the control group C (M) with the TCDD (M) group showed a statistically significant difference only in the $3^{\text {rd }}$ measurement, in which the response time in animals treated with a dioxin was extended. In TCDD (F) group, the extension of subsequent response times was observed (Fig. 1), where the $3^{\text {rd }}$ measurement revealed a significant statistical difference in relation to the $1^{\text {st }}$ measurement. The similar dynamics was noted in TCDD (M) group, however, with no statistically significant difference (Tables 1-3). A comparison between the obtained times from different groups TCDD (F) and TCDD (M) showed no statistically significant differences (Fig. 1).

The conclusion after comparing the response times of TCDD (F) group to TCDD + TCP (F) and TCDD + ASA (F) groups was that all response times in these groups were shortened, but a statistically significant difference occurred only in the $3^{\text {rd }}$ measurement in both groups. The conclusion after comparing the response time of TCDD (F) group to TCDD + TCP + ASA (F) group was that all response times in these groups were shortened; however, a statistically significant difference occurred in the $2^{\text {nd }}$ and $3^{\text {rd }}$ measurement in both groups. The conclusion after comparing the response times of TCDD + $\mathrm{TCP}+\mathrm{ASA}(\mathrm{F})$ group to TCDD + TCP (F) and TCDD + ASA (F) groups was that a statistically significant reduction in response time was observed in the $1^{\text {st }}$ measurement for TCDD + TCP (F) group. Comparing the response time of $\mathrm{K}(\mathrm{F})$ group to the response times of TCP $(\mathrm{F})$

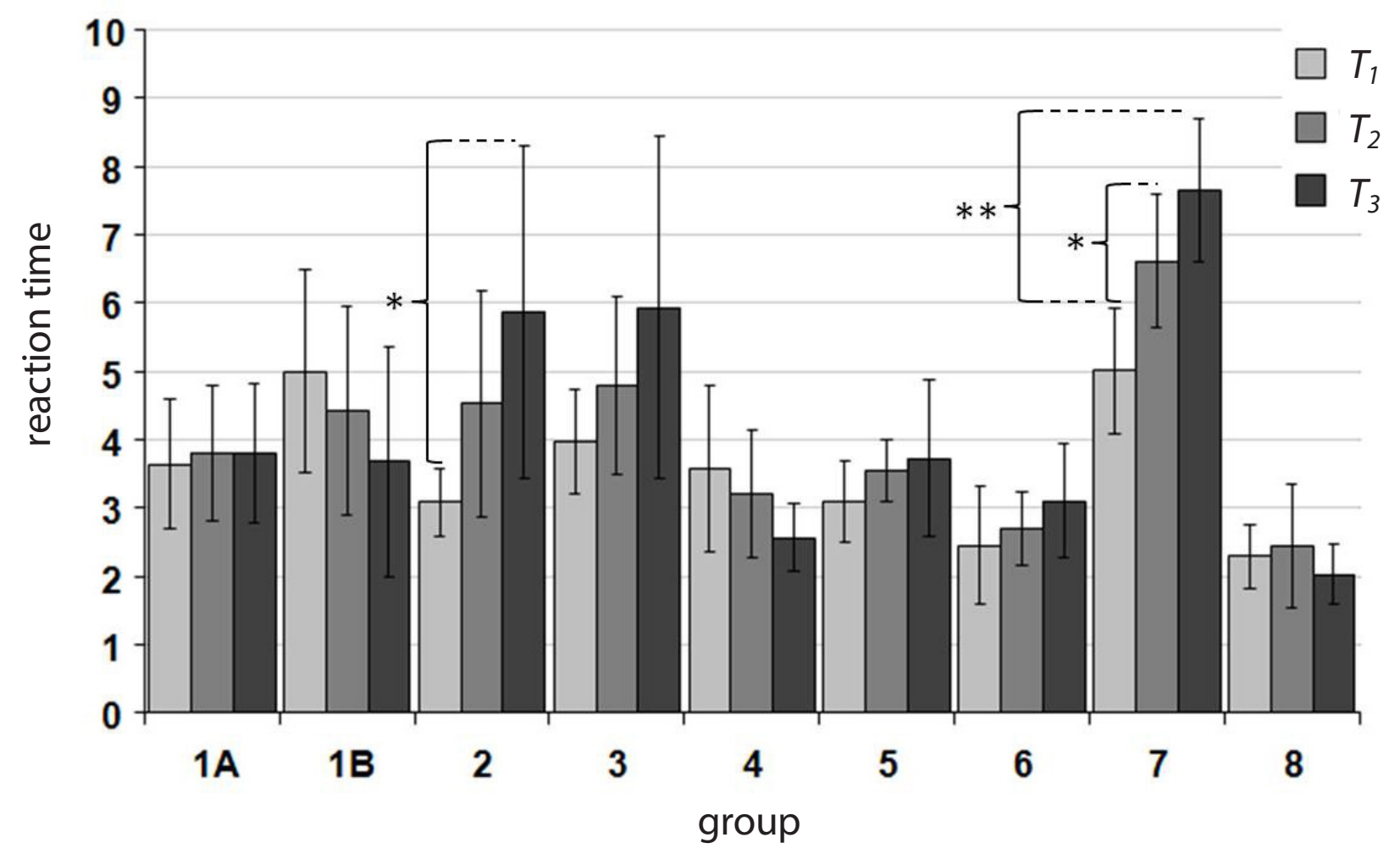

Fig. 1. Response times [s] of 3 subsequent measurements in particular groups in the tail test 
Table 1. Significance of differences between the groups in the $T_{1}$ measurement of the tail test ( $p$-values)

\begin{tabular}{|c|c|c|c|c|c|c|c|c|c|}
\hline Study group & $1 \mathrm{~A}$ & $1 \mathrm{~B}$ & $\mathbf{2}$ & $\mathbf{3}$ & 4 & 5 & 6 & 7 \\
\hline $1 \mathrm{~A}$ & - & 0.013 & 0.297 & 0.518 & 0.904 & 0.330 & 0.037 & 0.012 & 0.009 \\
\hline $1 \mathrm{~B}$ & 0.013 & - & 0.001 & 0.059 & 0.010 & 0.001 & 0.000 & 0.985 & 0.000 \\
\hline 2 & 0.297 & 0.001 & - & 0.095 & 0.356 & 0.983 & 0.258 & 0.001 & 0.114 \\
\hline 3 & 0.518 & 0.059 & 0.095 & - & 0.444 & 0.116 & 0.008 & 0.057 & 0.001 \\
\hline 4 & 0.904 & 0.010 & 0.356 & 0.444 & - & 0.390 & 0.048 & 0.009 & 0.012 \\
\hline 5 & 0.330 & 0.001 & 0.983 & 0.116 & 0.390 & - & 0.270 & 0.001 & 0.128 \\
\hline 7 & 0.037 & 0.000 & 0.258 & 0.008 & 0.048 & 0.270 & - & 0.000 & 0.756 \\
\hline 8 & 0.012 & 0.985 & 0.001 & 0.057 & 0.009 & 0.001 & 0.000 & - \\
\hline
\end{tabular}

Fisher's least significant difference post hoc test; $p<0.05$.

Table 2. Significance of differences between the groups in the $T_{2}$ measurement of the tail test ( $p$-values)

\begin{tabular}{|c|c|c|c|c|c|c|c|c|c|}
\hline Study group & $1 \mathrm{~A}$ & $1 \mathrm{~B}$ & $\mathbf{2}$ & 3 & 4 & 5 & 6 & 7 \\
\hline $1 \mathrm{~A}$ & - & 0.336 & 0.266 & 0.131 & 0.352 & 0.705 & 0.108 & 0.000 & 0.028 \\
\hline $1 \mathrm{~B}$ & 0.336 & - & 0.878 & 0.574 & 0.062 & 0.198 & 0.014 & 0.001 & 0.002 \\
\hline 2 & 0.266 & 0.878 & - & 0.682 & 0.044 & 0.153 & 0.009 & 0.002 & 0.001 \\
\hline 3 & 0.131 & 0.574 & 0.682 & - & 0.017 & 0.071 & 0.003 & 0.006 & 0.000 \\
\hline 4 & 0.352 & 0.062 & 0.044 & 0.017 & - & 0.608 & 0.462 & 0.000 & 0.210 \\
\hline 5 & 0.705 & 0.198 & 0.153 & 0.071 & 0.608 & - & 0.234 & 0.000 & 0.086 \\
\hline 7 & 0.108 & 0.014 & 0.009 & 0.003 & 0.462 & 0.234 & - & 0.000 & 0.680 \\
\hline 8 & 0.000 & 0.001 & 0.002 & 0.006 & 0.000 & 0.000 & 0.000 & - \\
\hline
\end{tabular}

Fisher's least significant difference post hoc test; $p<0.05$.

Table 3. Significance of differences between the groups in the $T_{3}$ measurement of the tail test ( $p$-values)

\begin{tabular}{|c|c|c|c|c|c|c|c|c|c|}
\hline Study group & $1 \mathrm{~A}$ & $1 \mathrm{~B}$ & $\mathbf{2}$ & $\mathbf{3}$ & 4 & 5 & 6 & 7 & 8 \\
\hline $1 \mathrm{~A}$ & - & 0.883 & 0.018 & 0.015 & 0.151 & 0.934 & 0.436 & 0.000 & 0.030 \\
\hline 1B & 0.883 & - & 0.012 & 0.010 & 0.196 & 0.954 & 0.522 & 0.000 & 0.043 \\
\hline 2 & 0.018 & 0.012 & - & 0.945 & 0.000 & 0.019 & 0.003 & 0.042 & 0.000 \\
\hline 3 & 0.015 & 0.010 & 0.945 & - & 0.000 & 0.016 & 0.003 & 0.049 & 0.000 \\
\hline 4 & 0.151 & 0.196 & 0.000 & 0.000 & - & 0.196 & 0.546 & 0.000 & 0.498 \\
\hline 5 & 0.934 & 0.954 & 0.019 & 0.016 & 0.196 & - & 0.504 & 0.000 & 0.047 \\
\hline 7 & 0.436 & 0.522 & 0.003 & 0.003 & 0.546 & 0.504 & - & 0.000 & 0.203 \\
\hline 8 & 0.000 & 0.000 & 0.042 & 0.049 & 0.000 & 0.000 & 0.000 & - \\
\hline
\end{tabular}

Fisher's least significant difference post hoc test; $p<0.05$.

group, a statistically significant increase in 3 response times in a group TCP (F) was observed both in relation to the control group $C(F)$ and subsequent measurements in this group (Fig. 1; Tables 1-6). A statistically significant reduction in all response times in ASA (F) group in comparison to the control group C (F) was observed (Fig. 3). On the other hand, a statistically significant increase in all response times in TCP (F) group in comparison to the control group C (F) was observed (Fig. 4).

\section{Results of the tail test in offspring groups}

The analysis of response times was conducted with the use of the tail test on 6 weeks old female offspring born to females treated with dioxins: TCDD (F) group, the group in which TCP was used: TCP + TCDD (F), and the group also treated with ASA: TCDD + ASA (F).

The offspring response time in the tail test in PTCDD group in the $2^{\text {nd }}$ measurement was significantly extended 
and characterized by a high standard deviation, however PTCDD + TCP, and PTCDD + ASA and PTCDD + TCP + ASA were characterized by a reduced response time in 3 subsequent measurements, which statistically differed significantly from the control group C and PTCDD (Tables 4-6; Fig. 5, 6). The corresponding groups of females which gave birth to the studied offspring TCDD + TCP (F), TCDD + ASA (F) and TCDD + TCP + ASA (F) in the $2^{\text {nd }}$ and $3^{\text {rd }}$ measurement of response times its reduction was observed, similarly to the response of their offspring described above (Fig. 5, 6). The response time in $T 1$ offspring of rats from the control group $\mathrm{C}$ and PTCDD group is significantly longer than in other groups $(\mathrm{p}<0.001)$. The response time in $T 2$ offspring of the rats from the control group $C$ and PTCDD group is significantly longer than in other groups ( $\mathrm{p}<0.05$ ). The response time T3 offspring of the rats from the control group $\mathrm{C}$ and PTCDD group is significantly longer than in other groups $(\mathrm{p}<0.05)$.

Analyzing the response time, based on the tail test, in the offspring born to females exposed to TCDD which were given ASA (PTCDD + ASA), for 3 weeks prior to pregnancy, similarly to adult females from this group, a statistically significant reduction of response time was found in each of the 3 measurements in relation to the offspring group born to females treated with TCDD only. This response is similar to that observed in the offspring group born to females from TCDD + TCP group.

The analysis of the combined use of ASA and TCP in the tail test on animals exposed to TCDD, which induced the inflammatory reaction, showed a reduction in response times at the $2^{\text {nd }}$ and $3^{\text {rd }}$ measurement in relation to TCDD (F) group. As to TCDD + TCP (F) group, the response time was reduced in the $1^{\text {st }}$ measurement, but a general conclusion after analyzing the response times in this group is that they were shorter than in TCDD + TCP (F) and TCDD + ASA (F) groups. The offspring group of females exposed to TCDD, which were treated with TCP and ASA before pregnancy, also demonstrated a reduction of 3 response times in relation to all studied offspring groups. The swimming test in TCDD + TCP + ASA (F) group showed that, at the beginning, the swimming was accelerated and the immobility period in this group was significantly longer than in TCDD + TCP (F) and TCDD + ASA (F) groups. The immobility period, however, was shorter and intensity of swimming was higher compared to TCDD (F) group. male

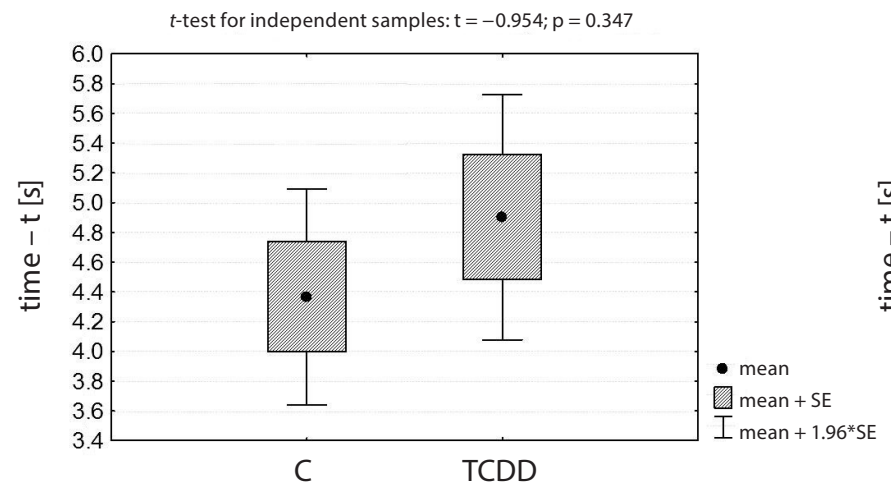

female

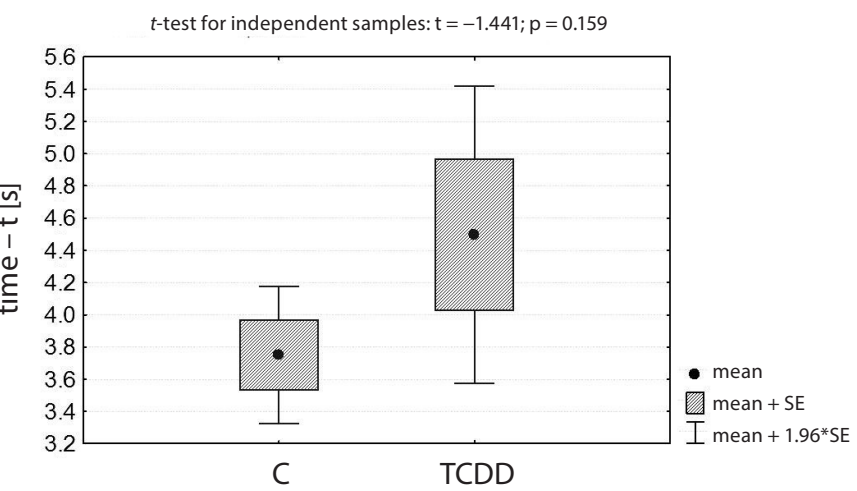

Fig. 2. Statistical analysis of mean response times [s] obtained between the groups of males and females from the control group and after TCDD administration

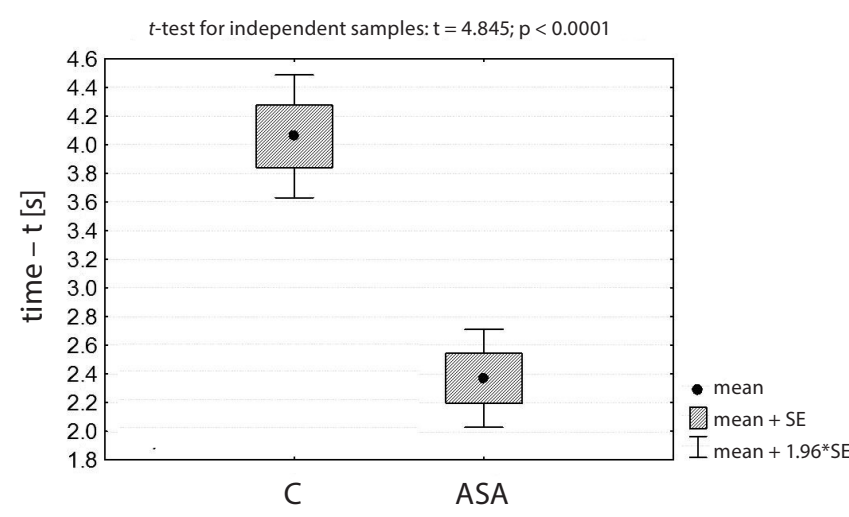

Fig. 3. Statistical analysis of mean response times [s] obtained between the control group C (F) and ASA (F)

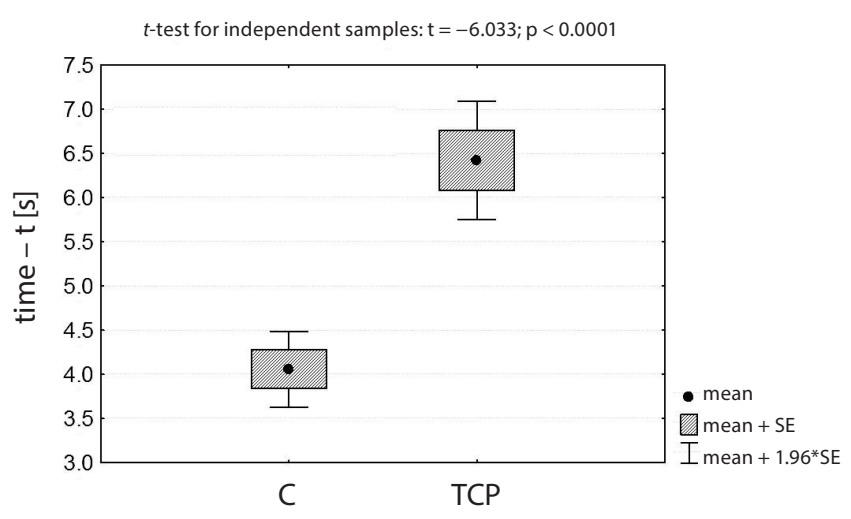

Fig. 4. Statistical analysis of mean response times [s] obtained between the control group C (F) TCP (F) 


\section{Results of the swimming test}

The swimming test in the control group $\mathrm{C}(\mathrm{F})$ and $(\mathrm{M})$ showed a statistically significant difference between males and females, finding an extended immobility period in females. In animals treated with dioxins, the comparison between a group of TCDD males (M) and TCDD females $(F)$ indicates statistically significant differences. Males from this group TCDD (M) did not make attempts to swim, showing a tendency to drown. In females from TCDD (F) group, the immobility time was significantly extended in relation to TCDD + TCP (F) and TCDD + ASA (F) groups. The results in this group showed a greater degree of deviation from the mean value in relation to the control group $\mathrm{C}(\mathrm{M})$. Analyzing the swimming test results in TCDD + ASA (F) group, there was no period of immobility observed and the swimming activity was intensified. The comparison of the control group $C(F)$ to ASA (F) group and TCP (F) shows a statistically significant reduction in immobility periods in TCP (F) and ASA (F) groups. The swimming time in TCDD + TCP + ASA (F) group was reduced in relation to TCDD (F) group (Table 6; Fig. 7, 8).

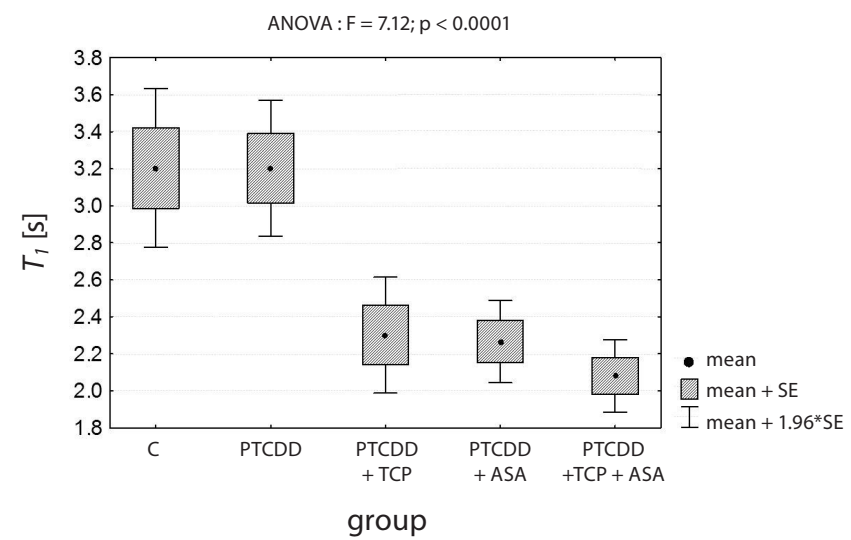

Fig. 5. Comparison of response times [s] from particular groups in the $T_{1}$ measurement

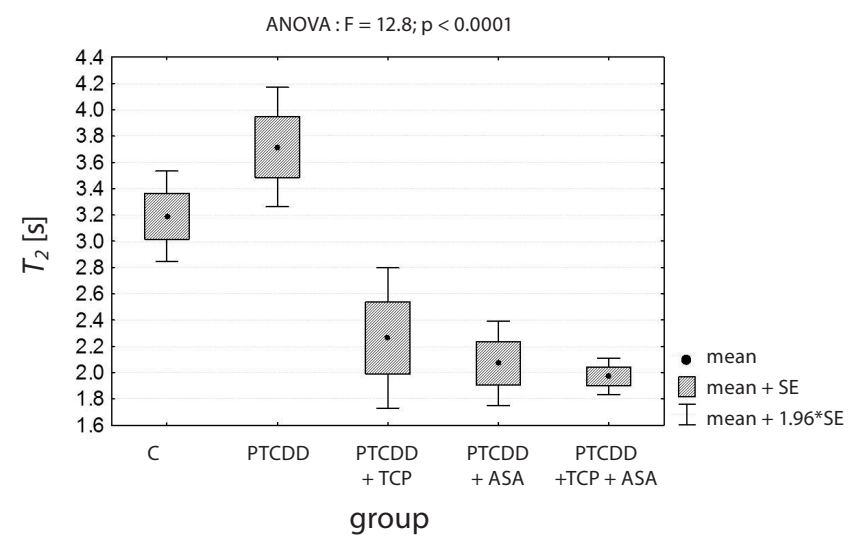

Fig. 6. Comparison of response times [s] from particular groups in the $T_{2}$ measurement
The evaluation of rats swimming intensity in a performed test showed that the control group $\mathrm{C}(\mathrm{F})$ was characterized by moderate intensity, while in $\mathrm{K}(\mathrm{M})$ group, the response was diversified (Table 6). In animals treated with dioxins, TCDD (F) group demonstrated a weak and moderate response, while in TCDD (M) group, there was no swimming response. In TCDD + ASA (F) group and TCDD + TCP + ASA (F) group the swimming response was intensified in relation to TCDD (F) group, in TCDD + TCP (F) group the response was moderate with a slight tendency to intensify. Comparing the control group C (F) to ASA (F) group, a significant intensification of response was observed after ASA administration. Administration of TCP in TCP group (F) induced weaker dynamics of response (Table 6).

\section{Discussion}

In our study, behavioral changes were observed in rats exposed to dioxins in terms of reduced physical activity, apathy and reduced food intake, often leading to cachexia and reduction in the concentration of plasma proteins. These findings are confirmed by studies of other authors. ${ }^{41-43}$

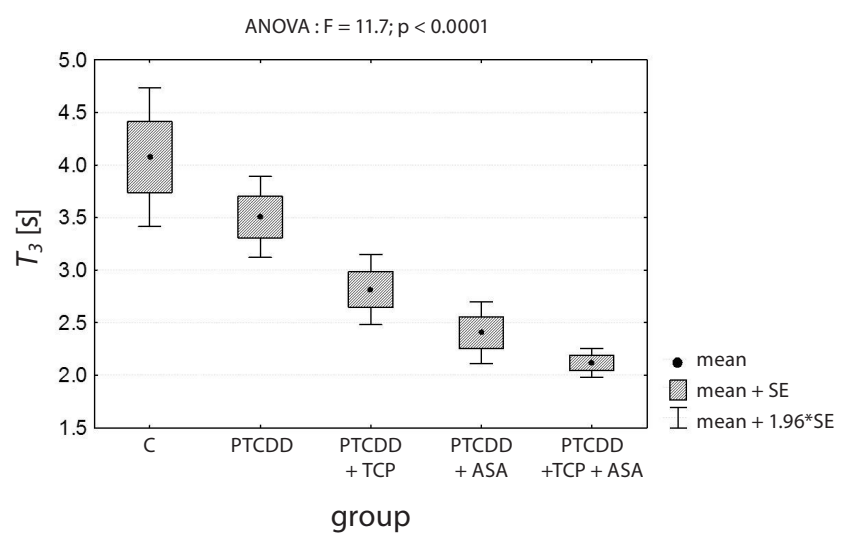

Fig. 7. Comparison of response times [s] from particular groups in the $T_{3}$ measurement

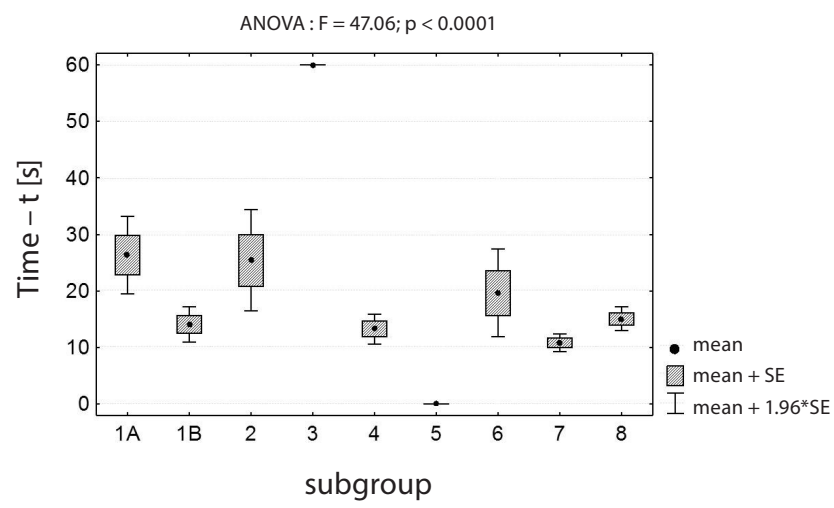

Fig. 8. Dynamics of the measured immobility time [s] of rats in the swimming test between particular groups 
This type of behavior was also observed in the offspring of rats born to females treated with TCDD. ${ }^{18,20}$ During the observation of offspring of both sexes born to females exposed to TCDD, males did not demonstrate any attempt to swim, whereas in females, the immobility time was significantly extended. This behavior supports the occurrence of depressive changes, and is associated with the lack of motivation to swim. Assessing the response times from the tail test in the animals treated with dioxins in relation to the control group, it was demonstrated that the response time was extended in the $3^{\text {rd }}$ measurement in both females and males, which is the indicator of the extension of time and route of nerve impulses conduction. This is also supported by the extension of subsequent response times. Both reflex and behavioral function disorders should be explained by the formation of free radicals affecting the CNS and higher levels of pro-inflammatory interleukins (IL-1 and II-6), and especially TNF, whose increased concentration in inflammation induces appetite disorders and apathy which was proven in numerous studies. ${ }^{2,42,44,45}$

Table 4. Significance of differences in response times from the $T_{1}$ measurement in the offspring born to females from different study groups ( $p$-values)

\begin{tabular}{|l|c|c|c|c|c|}
\multicolumn{1}{|c|}{ Study group } & $\begin{array}{c}\{1\} \\
=3.20\end{array}$ & $\begin{array}{c}\{2\} \\
=3.20\end{array}$ & $\begin{array}{c}\{3\} \\
=2.30\end{array}$ & $\begin{array}{c}\{4\} \\
=2.27\end{array}$ & $\begin{array}{c}\{5\} \\
=2.08\end{array}$ \\
\hline$\{1\} C(F)$ & - & 0.996 & 0.004 & 0.001 & 0.001 \\
\hline$\{2\}$ PTCDD & 0.996 & - & 0.004 & 0.001 & 0.001 \\
$\{3\}$ PTCDD + TCP & 0.004 & 0.004 & - & 0.913 & 0.566 \\
\hline$\{4\}$ PTCDD + ASA & 0.001 & 0.001 & 0.913 & - & 0.603 \\
\hline$\{5\}$ PTCDD + TCP + ASA & 0.001 & 0.001 & 0.566 & 0.603 & - \\
\hline
\end{tabular}

Fisher's least significant difference post hoc test; $p<0.05$.

Table 5. Significance of differences in response times from the $T_{2}$ measurement in the offspring born to females from different study groups ( $p$-values)

\begin{tabular}{|l|c|c|c|c|c|}
\multicolumn{1}{|c|}{ Study group } & $\begin{array}{c}\{1\} \\
=3.19\end{array}$ & $\begin{array}{c}\{2\} \\
=3.51\end{array}$ & $\begin{array}{c}\{3\} \\
=2.82\end{array}$ & $\begin{array}{c}\{4\} \\
=2.40\end{array}$ & $\begin{array}{c}\{5\} \\
=2.12\end{array}$ \\
\hline$\{1\} C(F)$ & - & 0.143 & 0.027 & 0.005 & 0.007 \\
$\{2\}$ PTCDD & 0.143 & - & 0.000 & 0.000 & 0.000 \\
$\{3\}$ PTCDD + TCP & 0.027 & 0.000 & - & 0.576 & 0.474 \\
$\{4\}$ PTCDD + ASA & 0.005 & 0.000 & 0.576 & - & 0.793 \\
\hline 5 PTCDD + TCP + ASA & 0.007 & 0.000 & 0.474 & 0.793 & - \\
\hline
\end{tabular}

Fisher's least significant difference post hoc test; $\mathrm{p}<0.05$.

Table 6. Significance of differences in response times from the $T_{3}$ measurement in the offspring born to females from different study groups ( $p$-values)

\begin{tabular}{|l|c|c|c|c|c|}
\multicolumn{1}{|c|}{ Study group } & $\begin{array}{c}\{1\} \\
=4.08\end{array}$ & $\begin{array}{c}\{2\} \\
=3.51\end{array}$ & $\begin{array}{c}\{3\} \\
=2.82\end{array}$ & $\begin{array}{c}\{4\} \\
=2.40\end{array}$ & $\begin{array}{c}\{5\} \\
=2.12\end{array}$ \\
\hline$\{1\} C(F)$ & - & 0.076 & 0.001 & 0.000 & 0.000 \\
\hline$\{2\}$ PTCDD & 0.076 & - & 0.019 & 0.000 & 0.000 \\
\hline$\{3\}$ PTCDD + TCP & 0.001 & 0.019 & - & 0.183 & 0.059 \\
$\{4\}$ PTCDD + ASA & 0.000 & 0.000 & 0.183 & - & 0.395 \\
\hline$\{5\}$ PTCDD + TCP + ASA & 0.000 & 0.000 & 0.059 & 0.395 & - \\
\hline
\end{tabular}

Fisher's least significant difference post hoc test; $p<0.05$.
Unusual behavior resulting from the influence that dioxins exert on the body, comparable with the results obtained in our study, was found in polar bears. Studies by Sonne et al. and Verreault et al. revealed that the cause of numerous disorders in the reproductive tract of both sexes is a significant concentration of dioxins accumulated in the bodies of mammals. ${ }^{46,47}$ Many studies found that estrogens affect cognition, memory, mood, behavior, emotional reactions, pain, posture, balance, and movement. ${ }^{7}$ In view of the above, it should be believed that the unusual behavior of people intoxicated with dioxins (rats in this study, studies on polar bears) is associated with disturbances in the metabolism of estrogen, as it was also observed in the studies by other authors. ${ }^{27,28,48,49}$

The evaluation of response time of animals treated with dioxins and TCP: TCDD + TCP (F) showed the reduction in the response time in the tail test in relation to the group which was given only TCDD (F). Measurements of the 3 subsequent response times in TCDD + TCP (F) group demonstrated a reduction of all 3 times against each other, implying the improvement of reflex responses.

Measurements of response times in the tail test of an offspring 6 weeks of age born to females exposed to TCDD (PTCDD $+\mathrm{TCP})$, which were given TCP for 3 weeks before pregnancy, also showed the same change trends as their mother, which demonstrated a statistically significant reduction in responses in all 3 measurements.

These observations suggest that TCP administered to females before pregnancy induces lower intoxication of their offspring with this dioxin from mothers, and less damage to the CNS in the offspring.

Similarly, the swimming test in female subjects exposed to TCDD and treated with TCP showed, in TCDD groups, in terms of intensity level, a significant reduction in the immobility period, which proves the improvement of reflex reaction and reduction of depressive reaction. The improving effect obtained after a long-term use of TCP in both tests can be justified by a protective role of this vitamin on the phospholipids of cell membranes, mitochondrial membranes, as well as reduced activity of free radicals and nitric oxide, and lower level of TNF concentration, which is also confirmed by studies of other authors. $^{50-52}$

Administration of ASA in ASA (F) group also resulted in shorter response times, which confirms the positive effect of ASA on neurotransmission. Similar results are presented in the studies by Gong et al., Wang and $\mathrm{Lu}$ et al. ${ }^{40,53,54}$ This study confirms 
that ASA is an antagonist of the glycine receptor and it reduces the inhibitory effect of GABA, releases glutamate from presynaptic terminals, resulting in the facilitation effect, which induces the hyperreactivity. This can be beneficial both in subjects with delayed response times and depressive type disorders that occur in dioxin intoxication, as has been manifested in the swimming test with no attempts to swim or with extended immobility in swimming.

The obtained response time results suggest that the administration of ASA and TCP in mothers treated with TCDD before pregnancy eliminated the negative effects of dioxin on CNS functioning in offspring compared to the offspring born to the females treated with TCDD only.

The analysis of the swimming test in rats exposed to dioxins, which are treated with ASA, showed a lack of immobility period and intensified continuous swimming. This type of reaction also occurred in S (F) group. These results support the idea that ASA has a strong anti-inflammatory effect, eliminating the negative effects of the proinflammatory action of TCDD connected with generating a large number of free radicals, TNF and prostaglandins which affect the functioning of the CNS, induce depression, apathy and lethargy (Fig. 2). These findings can be confirmed by similar results obtained in studies by other authors. ${ }^{45,55-57}$ In addition, our most recent studies with the use of histopathological and ultrastructural analysis of the hippocampus in rats found that TCDD contributes to atrophy of estrogen receptors, in which also destructive and inflammatory changes were found along with demyelination of myelin sheaths. It was determined a total protective action of TCP and ASA towards CNS functions that was characterized by poorly expressed degenerative changes and smaller inflammatory reactivity. ${ }^{16}$

\section{Conclusions}

Dioxin has an angiotoxic, neurotoxic and glycotoxic effect, which induces behavioral disorders associated with prolonged response times. Administration of TCP causes a significant reduction and improvement of reflex response times, which is also confirmed in the offspring born to females exposed to TCDD and treated with TCP. The use of ASA also reduces the reflex response times in the offspring of females exposed to TCDD. Based on the results, it can be concluded that the combined use of TCP and ASA significantly reduces the response time in the tail test and increases the intensity of the swimming activity.

\section{References}

1. Crow KD. Chloracne and its potential clinical implications. Clin Exp Dermatol. 1981;6:243-257.

2. Arora S. Leptin and its metabolic interactions: An update. Diabetes Obes Metab. 2008;10:973-993.

3. Ciftci O, Vardi N, Ozdemir I. Effects of quercetin and chrysin on 2,3,7,8-tetrachlorodibenzo-p-dioxin induced hepatotoxicity in rats. Environ Toxicol. 2013;28(3):146-154.
4. Stevens EA, Mezrich JD, Bradfield CA. The aryl hydrocarbon receptor: A perspective on potential roles in the immune system. Immunology. 2009;127:299-311.

5. Stockinger B, Hirota K, Duarte J, Veldhoen M. External influences on the immune system via activation of the aryl hydrocarbon receptor. Semin Immunol. 2011;23:99-105.

6. Teraoka H, Kubota A, Dong W, et al. Role of the cyclooxygenase 2-thromboxane pathway in 2,3,7,8-tetrachlorodibenzo-p-dioxininduced decrease in mesencephalic vein blood flow in the zebrafish embryo. Toxicol Appl Pharmacol. 2009;234:33-40.

7. Clements RJ, Lawrence RC, Blank JL. Effects of intrauterine 2,3,7,8-tetrachlorodibenzo-p-dioxin on the development and function of the gonadotrophin releasing hormone neuronal system in the male rat. Reprod Toxicol. 2009;28:38-45.

8. Fukushima K, Tsukimori K, Li D, et al. Effect of transient TCDD exposure on immortalized human trophoblast-derived cell lines. Hum Exp Toxicol. 2012;31:550-556.

9. Fernández M, Paradisi M, D'Intino G, et al. A single prenatal exposure to the endocrine disruptor 2,3,7,8-tetrachlorodibenzo-p-dioxin alters developmental myelination and remyelination potential in the rat brain. J Neurochem. 2010;115:897-909.

10. Hood DB, Woods L, Brown L, Johnson S, Ebner FF. Gestational 2,3,7,8-tetrachlorodibenzo-p-dioxin exposure effects on sensory cortex function. Neurotoxicology. 2006;27:1032-1042.

11. Nishijo M, Kuriwaki Jl, Hori E, Tawara K, Nakagawa H, Nishijo H. Effects of maternal exposure to 2,3,7,8-tetrachlorodibenzo-p-dioxin on fetal brain growth and motor and behavioral development in offspring rats. Toxicol Lett. 2007;173:41-47.

12. Williamson MA, Gasiewicz TA, Opanashuk LA. Aryl hydrocarbon receptor expression and activity in cerebellar granule neuroblasts: Implications for development and dioxin neurotoxicity. Toxicol Sci. 2005;83:340-348.

13. Nishijo M, Tawara K, Nakagawa H, et al. 2,3,7,8-Tetrachlorodibenzop-dioxin in maternal breast milk and newborn head circumference. J Expo Sci Environ Epidemiol. 2008;18:246-251.

14. Andreasen $\mathrm{CH}$, Stender-Petersen KL, Mogensen MS, et al. Low physical activity accentuates the effect of the FTO rs9939609 polymorphism on body fat accumulation. Diabetes. 2008;57:95-101.

15. Dong B, Cheng W, Li W, et al. FRET analysis of protein tyrosine kinase c-Src activation mediated via aryl hydrocarbon receptor. Biochim Biophys Acta. 2011;1810:427-431.

16. Rosińczuk J, Dymarek R, Całkosiński I. Histopathological, ultrastructural, and immunohistochemical assessment of hippocampus structures of rats exposed to TCDD and high doses of tocopherol and acetylsalicylic acid. Bio Med Res Int. 2015;2015:645603.

17. Yoon CY, Park M, Kim BH, et al. Gene expression profile by 2,3,7,8-tetrachlorodibenzo-p-dioxin in the liver of wild-type (AhR+/+) and aryl hydrocarbon receptor-deficient (AhR-/-) mice. J Vet Med Sci. 2006;68:663-668.

18. Całkosiński I, Borodulin-Nadzieja L, Stańda M, Wasilewska U, Cegielski M. Influence of a single dose of TCDD on estrogen levels and reproduction in female rats. Vet Med. 2003;59:536-538.

19. Całkosiński I, Borodulin-Nadzieja L, Wasilewska U, et al. Effect of dioxins on reproduction in rats in vivo. Adv Clin Exp Med. 2004;13:885-890.

20. Całkosiński I, Wasilewska U, Borodulin-Nadzieja L, et al. Influence of 2,3,7,8-tetrachlorodibenzo-p-dioxin (TCDD) on the functioning and structure of ovaries and testicles in the offspring of rats. Vet Med. 2004;60:1218-1221.

21. Całkosiński I, Dobrzyński M, Całkosińska M, et al. Characterization of an inflammatory response. Adv Hyg Exp Med. 2009;63:395-408.

22. Wen S, Gong Y, Li J, Shi T, Zhao Y, Wu Y. Particle-bound PCDD/Fs in the atmosphere of an electronic waste dismantling area in China. Biomed Environ Sci. 2011;24:102-111.

23. Geusau A, Tschachler E, Meixner M, Päpke O, Stingl G, McLachlan M. Cutaneous elimination of 2,3,7,8-tetrachlorodibenzo-p-dioxin. Br J Dermatol. 2001;145:938-943.

24. Geusau A, Abraham K, Geissler K, Sator MO, Stingl G, Tschachler E. Severe 2,3,7,8-tetrachlorodibenzo-p-dioxin (TCDD) intoxication: Clinical and laboratory effects. Environ Health Perspect. 2001;109: 865-869. 
25. Cheshenko K, Brion F, Le Page Y, et al. Expression of zebra fish aromatase cyp19a and cyp19b genes in response to the ligands of estrogen receptor and aryl hydrocarbon receptor. Toxicol Sci. 2007;96:255-267.

26. Mukai M, Lin TM, Peterson RE, Cooke PS, Tischkau SA. Behavioral rhythmicity of mice lacking $A h R$ and attenuation of light-induced phase shift by 2,3,7,8-tetrachlorodibenzo-p-dioxin. J Biol Rhythms. 2008;23:200-210.

27. Oehme M, Biseth A, Schlabach M, Wiig $\varnothing$. Concentrations of polychlorinated dibenzo-p-dioxins, dibenzofurans and non-ortho substituted biphenyls in polar bear milk from Svalbard (Norway). Environ Pollut. 1995;90:401-407.

28. Oehme M, Schlabach M, Hummert K, Luckas B, Nordøy ES. Determination of levels of polychlorinated dibenzo-p-dioxins, dibenzofurans, biphenyls and pesticides in harp seals from the Greenland Sea. Sci Total Environ. 1995;162:75-91.

29. Carvalho PSM, Tillitt DE. 2,3,7,8-TCDD effects on visual structure and function in swim-up rainbow trout. Environ Sci Technol. 2004;38:6300-6306.

30. Tsukamoto H, Rippe R, Niemelä O, Lin M. Roles of oxidative stress in activation of Kupffer and Ito cells in liver fibrogenesis. J Gastroenterol Hepatol. 1995;10:50-53.

31. Tue NM, Suzuki G, Takahashi S, et al. Evaluation of dioxin-like activities in settled house dust from Vietnamese E-waste recycling sites: Relevance of polychlorinated/brominated dibenzo-p-dioxin/furans and dioxin-like PCBs. Environ Sci Technol. 2010;44:9195-9200.

32. Hassoun EA, Vodhanel J, Abushaban A. The modulatory effects of ellagic acid and vitamin E succinate on TCDD-induced oxidative stress in different brain regions of rats after subchronic exposure. J Biochem Mol Toxicol. 2004;18:196-203.

33. Hassoun EA, Vodhanel J, Holden B, Abushaban A. The effects of ellag ic acid and vitamin $E$ succinate on antioxidant enzymes activities and glutathione levels in different brain regions of rats after subchronic exposure to TCDD. J Toxicol Environ Health A. 2006;69:381-393.

34. Kim SY, Lee HG, Choi EJ, Park KY, Yang JH. TCDD alters PKC signaling pathways in developing neuronal cells in culture. Chemosphere. 2007;67:421-427.

35. Kim SY, Yang JH. Neurotoxic effects of 2,3,7,8-tetrachlorodibenzop-dioxin in cerebellar granule cells. Exp Mol Med. 2005;37:58-64.

36. Li W, Matsumura F. Significance of the nongenomic, inflammatory pathway in mediating the toxic action of TCDD to induce rapid and long-term cellular responses in 3T3-L1 adipocytes. Biochemistry (Mosc). 2008;47:13997-4008.

37. MacDonald CJ, Cheng RYS, Roberts DD, Wink DA, Yeh GC. Modulation of carcinogen metabolism by nitric oxide-aspirin 2 is associated with suppression of DNA damage and DNA adduct formation. J Biol Chem. 2009;284:22099-22107.

38. MacDonald CJ, Ciolino HP, Yeh GC. The drug salicylamide is an antagonist of the aryl hydrocarbon receptor that inhibits signal transduction induced by 2,3,7,8-tetrachlorodibenzo-p-dioxin. Cancer Res. 2004;64:429-434.

39. Maharaj H, Maharaj DS, Daya S. Acetylsalicylic acid and acetaminophen protect against oxidative neurotoxicity. Metab Brain Dis. 2006;21:189-199.

40. Wang SJ. Facilitatory effect of aspirin on glutamate release from rat hippocampal nerve terminals: Involvement of protein kinase $C$ pathway. Neurochem Int. 2006;48(3):181-190.

41. Całkosiński I. The influence of tocopherol on diagnostic indexes of inflammatory reaction in rats undergoing dioxin exposition [habilitation thesis]. Wrocław, Poland: Wroclaw Medical University; 2008.
42. Całkosiński l. The course of experimentally induced acute pleuritis with use of nitrogranulogen (NTG) and 2,3,7,8-tetrachlorodibenzo-p-dioxin (TCDD) [habilitation thesis]. Wrocław, Poland:Wroclaw Medical University; 2005.

43. Moon BH, Hong CG, Kim SY, et al. A single administration of 2,3,7,8-tetrachlorodibenzo-p-dioxin that produces reduced food and water intake induces long-lasting expression of corticotropin-releasing factor, arginine vasopressin, and proopiomelanocortin in rat brain. Toxicol Appl Pharmacol. 2008;233:314-422.

44. Lensu S, Miettinen R, Pohjanvirta R, Lindén J, Tuomisto J. Assessment by c-Fos immunostaining of changes in brain neural activity induced by 2,3,7,8-tetrachlorodibenzo-p-dioxin (TCDD) and leptin in rats. Basic Clin Pharmacol Toxicol. 2006;98:363-371.

45. Li X, Johnson DC, Rozman KK. Effects of 2,3,7,8-tetrachlorodibenzo-p-dioxin (TCDD) on estrous cyclicity and ovulation in female Sprague-Dawley rats. Toxicol Lett. 1995;78:219-222.

46. Sonne C, Leifsson PS, Dietz R, et al. Xenoendocrine pollutants may reduce size of sexual organs in East Greenland polar bears (Ursus maritimus). Environ Sci Technol. 2006;40:5668-5674.

47. Verreault J, Norstrom RJ, Ramsay MA, Mulvihill M, Letcher RJ. Composition of chlorinated hydrocarbon contaminants among major adipose tissue depots of polar bears (Ursus maritimus) from the Canadian high Arctic. Sci Total Environ. 2006;370:580-587.

48. Jin $\mathrm{MH}$, Hong $\mathrm{CH}$, Lee HY, Kang $\mathrm{HJ}$, Han SW. Enhanced TGF-beta1 is involved in 2,3,7,8-tetrachlorodibenzo-p-dioxin (TCDD) induced oxidative stress in C57BL/6 mouse testis. Toxicol Lett. 2008;178:202-209.

49. Kakeyama $M$, Sone $H$, Tohyama $C$. Perinatal exposure of female rats to $2,3,7,8$-tetrachlorodibenzo-p-dioxin induces central precocious puberty in the offspring. J Endocrinol. 2008;197:351-358.

50. Delwing D, Tagliari B, Chiarani F, Wannmacher CM, Wajner M, Wyse AT. Alpha-tocopherol and ascorbic acid administration prevents the impairment of brain energy metabolism of hyperargininemic rats. Cell Mol Neurobiol. 2006;26:177-189.

51. Kloser E, Böhmdorfer S, Brecker L, et al. Synthesis of 5-(fluorophenyl)tocopherols as novel dioxin receptor antagonists. Eur J Org Chem. 2011;2011:2450-2457.

52. Norazlina M, Lee PL, Lukman HI, Nazrun AS, Ima-Nirwana S. Effects of vitamin E supplementation on bone metabolism in nicotine-treated rats. Singapore Med J. 2007;48:195-199.

53. Gong N, Zhang M, Zhang X-B, Chen L, Sun GC, Xu TL. The aspirin metabolite salicylate enhances neuronal excitation in rat hippocampal CA1 area through reducing GABAergic inhibition. Neuropharmacology. 2008;54:454-463.

54. Lu YG, Tang ZQ, Ye ZY, et al. Salicylate, an aspirin metabolite, specifically inhibits the current mediated by glycine receptors containing alpha1-subunits. Br J Pharmacol. 2009;157:1514-1522.

55. Całkosiński I, Gamian A, Dobrzyński M. Possibilities of the use of tocopherol in the case of intoxication with dioxins. In: Żuber M, ed. Natural and Civilization Catastrophes, the dangers and challenges for the global gecurity. Wrocław: The Tadeusz Kościuszko Land Forces Military Academy Publishing House; 2009:275-284.

56. Fatokun AA, Stone TW, Smith RA. Cell death in rat cerebellar granule neurons induced by hydrogen peroxide in vitro: Mechanisms and protection by adenosine receptor ligands. Brain Res. 2007;1132:193-202.

57. Takada Y, Bhardwaj A, Potdar P, Aggarwal BB. Nonsteroidal antiinflammatory agents differ in their ability to suppress NF-kappaB activation, inhibition of expression of cyclooxygenase-2 and cyclin D1, and abrogation of tumor cell proliferation. Oncogene. 2004;23:9247-9258. 